\title{
ORIGINALBEITRÄGE
}

\author{
Rebecca Bondü, Laura Tampe, Katharina Menke
}

\section{Gemeinsamkeiten und Unterschiede von Amokläufen an Bildungseinrichtungen und islamistisch motivierten terroristischen Taten in westlichen Ländern 1999-2017}

\section{Zusammenfassung}

Terroristische Anschläge und Amokläufe an Bildungseinrichtungen erschüttern die Bevölkerung gleichermaßen. Beide zielen auf die Tötung anderer im öffentlichen Raum und erhalten aufgrund der negativen Folgen und des symbolischen Charakters häufig große mediale Aufmerksamkeit. Die Täter sind meist junge Männer, so dass auch hinsichtlich der Täter scheinbar Ähnlichkeiten bestehen. Gemeinsamkeiten und Unterschiede von Tat- und Tätermerkmalen wurden bislang aber kaum untersucht. Wir verglichen 172 Amokläufe an Schulen, 30 Amokläufe an höheren Bildungseinrichtungen und 86 islamistisch motivierte terroristische Taten in sieben westlichen Ländern zwischen 1999 und 2017 hinsichtlich objektiver Tat- und Tätermerkmale. Ähnlichkeiten bestanden in der Geschlechtsverteilung und der Häufigkeit der Verwendung von Schusswaffen. Unterschiede zeigten sich in Bezug auf das Alter und den Migrationshintergrund der Täter, die Häufigkeit der Verwendung von Klingenwaffen und Bomben, der Anzahl der Täter, dem Tatzeitpunkt sowie der Anzahl an Toten und Verletzten. Die Studie zeigt somit sowohl Ähnlichkeiten als auch Unterschiede in den Tatund Tätermerkmalen. Unterschiede sind vermutlich durch situative Einflüsse und das Alter der Täter, welches den Zugang zu Waffen beeinflusst, bedingt, und haben wichtige Implikationen für präventive Ansätze.

Schlüsselwörter: Terrorismus, Amokläufe an Bildungseinrichtungen, Tatmerkmale, Tätereigenschaften 


\section{Similarities and Differences between Rampage in Educational Settings and Terroristic Acts in Western Countries 1999-2017}

\section{Abstract}

Terrorist attacks and school shootings are equally shocking. Both aim at killing others in public and gain intense media attention due to their negative consequences and strong symbolic character. The perpetrators are mostly young men, indicating that both offender groups may be similar. However, similarities and differences in offense and offender characteristics have hardly been examined. We compared 172 school attacks, 30 attacks in institutes of higher education, and 86 islamistic terrorist attacks in seven western countries between 1999 and 2017 with regard to objective offense and offender characteristics. We found similarities in gender and the frequency of firearm usage. There were differences in age and offender migration background, the use of bladed weapons and bombs, the number of offenders, the time of the attacks, and the number of dead and injured victims. Thus, the study shows similarities and differences in offense and offender characteristics. These are presumably due to situational influences and offender age, affecting access to weapons. These findings have important implications for preventive approaches.

Keywords: terrorism, rampage at educational institutions, offense characteristics, offender characteristics

\section{Theoretischer Hintergrund}

In den letzten Jahren wird zunehmend oft über islamistisch motivierte Terroranschläge berichtet. Ein Vergleich mit Amoktaten in Bildungseinrichtungen kann zeigen, ob Ansätze zu deren Prävention auf Terroranschläge übertragbar sind.

Terroristen und Amokläufer haben scheinbar sehr unterschiedliche Motive: Die einen scheinen ideologisch, die anderen persönlich motiviert (Bannenberg, 2017). Die Unterscheidung ist jedoch nicht immer einfach und trennscharf, denn beide Tätergruppen handeln zudem meist aus Rache und einem Gefühl der Benachteiligung (McCauley, Moskalenko \& Van Son, 2013). Auch aus der Tat lässt sich das Motiv nicht immer ableiten: So ist das Tatmotiv des Amoklaufs in München 2016 weiterhin umstritten. Generell ist von einer Vermengung persönlicher und (vermeintlich) ideologischer Motive auszugehen. So mehren sich die Hinweise, dass die Richtung einer Radikalisierung auch davon abhängt, ob eine und welche Ideologie verfügbar ist (Neumann, 2017).

Unterschiede in Motiven mögen Unterschiede in der Tat (z.B. die Auswahl von Opfern oder Ort) begründen. Doch auch in den Tatabläufen gibt es Überschneidungen zwischen Terroranschlägen und Amokläufen: So weitete sich der Amoklauf in Winnenden 2009 auf Orte außerhalb der Schule aus. Zudem zielen beide (häufig) auf eine symbolische Tötung vieler Menschen ab (Böckler, Leuschner, Zick \& Scheithauer, 2018), sind als Massenmorde zu betrachten und ähneln sich im modus operandi (z.B. langfristige Planung; McCauley et al., 2013). 
Somit ist nicht ausgemacht, ob zwischen islamistisch motivierten Tätern und Amokläufern eher Gemeinsamkeiten oder Unterschiede bestehen; empirische, vergleichende Forschung existiert kaum. Wir untersuchten daher Gemeinsamkeiten und Unterschiede in Tat- und Tätermerkmalen von Amokläufen im Bildungskontext und islamistisch motivierten Terroranschlägen in sieben westlichen Ländern zwischen 1999 und 2017.

\section{Definition von Terroranschlägen und von Amokläufen im Bildungskontext}

Für Terroranschläge und Amokläufe an Bildungseinrichtungen gibt es keine einheitlichen Definitionen. Wir definierten Terrorismus gemäß der Global Terrorism Database als "the [...] use of illegal force and violence by a non-state actor to attain a political, economic, religious, or social goal through fear, coercion, or intimidation" (START 2017b). Amokläufe an Bildungseinrichtungen wurden in Anlehnung an Bondü (2012) als gezielte Angriffe eines (ehemaligen) Schülers gegen mit der Schule assoziierte, zumindest teilweise zuvor ausgewählte Personen(gruppen) mit potentiell tödlichen Waffen und Tötungsabsicht definiert. Die Schule musste bewusst als Tatort gewählt sein und mit den individuell konstruierten Motiven des Täters in Zusammenhang stehen. Bei Amokläufen in Bildungseinrichtungen wurde zwischen Taten an Primar- und Sekundarschulen (z.B. Grundschulen, High-Schools) und höheren Bildungseinrichtungen (z.B. Colleges, Universitäten, Berufsschulen) unterschieden, da frühere Forschung Unterschiede in objektiven Täter- und Tatmerkmalen zwischen diesen zeigte (s.u., Bondü \& Beier, 2014).

Das Fehlen einheitlicher Definitionen erschwert es, Tathäufigkeiten zu beziffern, zumal Terroranschläge oder Amokläufe in Kriminalitätsstatistiken keine eigenen Tatbestände darstellen. Es sind aber jeweils seltene Phänomene: Bondü und Beier (2014) recherchierten für den Zeitraum von 1886 bis 2012 weltweit 232 Amokläufe an Schulen sowie 45 an höheren Bildungseinrichtungen. Während deren Häufigkeit in den letzten Jahren rückläufig war, haben terroristische Taten zugenommen. Zwischen 2002 und 2015 gab es weltweit 4900 islamistisch motivierte Terroranschläge. Im Jahr 2013 verübte allein der IS weltweit durchschnittlich 46 Anschläge pro Monat, 2014 waren es 106 pro Monat (Miller, Kane, Kammerer \& Wingenroth, 2016). Weltweit ereignen sich die meisten Terroranschläge in asiatischen und afrikanischen Ländern. Unter den westlichen Staaten waren die USA am häufigsten betroffen (Miller et al., 2016; Böckler, Seeger, Sitzer \& Heitmeyer, 2012).

Tat- und Tätermerkmale von Terroranschlägen und von Amokläufen im Bildungskontext

Bislang existiert lediglich eine Studie, die islamistisch motivierte Taten und Amokläufe an Bildungseinrichtungen vergleicht. Mittels Aktenanalyse von je sieben islamistischen Tätern und Amokläufern an Schulen konnte festgestellt werden, dass es in dem Entwicklungsprozess hin zu einer zielgerichteten Tat Überschneidungen gibt (z.B. symbo- 
lische Bedeutung; Böckler et al., 2018). Des Weiteren gibt es Vergleiche von Amokläufern in den USA und Selbstmordattentätern im Nahen Osten (Lankford \& Hakim, 2011) sowie terroristischen Einzeltätern/Amokläufern und nichtideologischen Mördern (Liem, van Buuren, de Roy van Zuijdewijn, Schönberger \& Bakker, 2018). Eine Studie verglich Amokläufer an Schulen und ideologisch motivierte Attentäter in den USA in Bezug auf demographische Charakteristika, Motivation und Verhalten vor und während der Tat (Capellan, Johnson, Porter \& Martin, 2018). Sie zeigte, dass Amokläufer und Attentäter meist männlich sind. Die Amokläufer waren im Schnitt jünger und töteten weniger Menschen und wurden eher festgenommen als getötet. Die Anzahl der Schusswaffen unterschied sich nicht. Weitere Studien untersuchten Gewalttaten an Bildungseinrichtungen und islamistisch motivierte terroristische Taten separat. Diese erlauben einen Vergleich der Befunde und lassen in folgenden Bereichen Unterschiede erwarten:

Alter. Islamistisch motivierte Terroristen (IMT) waren im Schnitt 28 (Gruenewald, Klein, Freilich \& Chermak, 2016), Amokläufer an höheren Bildungseinrichtungen (AHB) 30 Jahre alt; das Durchschnittsalter von Amokläufern an Schulen (AS) lag bei knapp 16 Jahren (Bondü \& Beier, 2014).

Migrationshintergrund. Die meisten IMT hatten einen Migrationshintergrund (Bakker, 2011), überwiegend aus Nordafrika, Pakistan oder Irak. Bei nur 23 Tätern der 65 Fälle islamistischen Terrors in Europa mit mindestens 336 involvierten Personen kamen die Familien ursprünglich aus Europa (Bakker, 2011). Etwa ein Drittel der AHB hatte einen Migrationshintergrund, überwiegend aus asiatischen Ländern; der Anteil der AS mit Migrationshintergrund lag dagegen bei nur 7\% (Bondü \& Beier, 2014).

Tatzeit. AS wurden meist im Frühling, Winter und Herbst ausgeführt (Bondü \& Beier, 2014); IMT waren über das Jahr fast gleichverteilt (START, 2016) und vermutlich weniger an spezifische Tageszeiten gebunden. Auch AHB zeigten keine klaren Häufungen (Bondü \& Beier, 2014). Sie geschahen überwiegend vormittags unter der Woche, insbesondere montags (Bondü \& Beier, 2014; Vossekuil, Fein, Reddy, Borum \& Modzeleski, 2002).

Tatwaffen. AHB verwendeten meist Schusswaffen als primäre Tatwaffe, andere Waffen waren selten; AS verwendeten aber häufiger Klingenwaffen als die an höheren Bildungseinrichtungen (Bondü \& Beier, 2014). IMT verwendeten am häufigsten Schusswaffen $(71.8 \%)$, aber auch explosive Stoffe $(5.1 \%)$ oder Fahrzeuge (12.8\%; Gruenewald et al., 2016).

Opfer. IMT wählten in 77\% der Fälle Opfer aus, zu denen keine persönliche Beziehung bestand (Gruenewald et al., 2016). Von AS waren überwiegend Schulpersonal oder Mitschüler betroffen (Bondü \& Beier, 2014), die den Tätern zumeist mindestens teilweise bekannt waren. Auch bei AHB waren meist Studierende und Schüler bzw. Lehrpersonal die Opfer (Bondü \& Beier, 2014). Ob die Täter die Opfer kannten, ist meist unbekannt. Meloy et al. (2004) zeigten, dass die Opferzahl erwachsener Massenmörder höher ist als die jugendlicher. Bei Anschlägen des IS starben im Mittel 7.5 Menschen (Miller et al., 2016). AHB forderten mehr Tote als AS, die Anzahl der Verletzten und aller Opfer unterschied sich nicht (Bondü \& Beier, 2014). 
Suizid. AHB (37.8\%) suizidierten sich häufiger als AS (14.2\%; Bondü \& Beier, 2014). Selbstmordanschläge machten weltweit etwa 19\% der Terroranschläge zwischen 1970 und 2015 aus (IEP, 2016).

Obwohl sich IMT und Amokläufer an Bildungseinrichtungen in vieler Hinsicht zu unterscheiden scheinen, finden sich auch Gemeinsamkeiten: Schusswaffen waren jeweils die häufigsten Tatwaffen, die Täter meist männlich (Bondü \& Beier, 2014; Gruenewald et al., 2016).

\section{Die aktuelle Studie}

Da entsprechende, systematische Studien bislang weitgehend fehlen, war das Ziel dieser Studie der Vergleich von IMT, AS und AHB hinsichtlich objektiver Tat- und Tätermerkmale anhand folgender Hypothesen:

Hypothese a: IMT pro Jahr haben in den betrachteten Staaten im Untersuchungszeitraum zwischen 1999 und 2017 zugenommen, AS und AHB abgenommen.

Hypothese b: Die meisten Taten haben in allen drei Gruppen in den USA stattgefunden.

Hypothese c: IMT sind älter als AS, nicht aber als AHB.

Hypothese d: Der Anteil der männlichen Täter unterscheidet sich nicht.

Hypothese e: IMT haben häufiger einen Migrationshintergrund als AS.

Hypothese f: IMT werden häufiger von Tätergruppen ausgeführt als AS und AHB, AS und AHB sollten demnach im Vergleich häufiger Einzeltäter sein.

Hypothese g: AS und AHB geschehen überwiegend im Frühling, Winter und Herbst, IMT über das Jahr verteilt.

Hypothese h: AS und AHB finden eher unter der Woche statt, IMT über die Woche verteilt.

Hypothese $i$ : AS und AHB geschehen eher vormittags, IMT zeigen keine zeitliche Häufung.

Hypothese j: Schusswaffen sind jeweils das häufigste Tatwerkzeug.

Hypothese k: IMT gebrauchen häufiger explosive Waffen als AS und AHB.

Hypothese l: $\quad$ IMT fordern mehr Tote und Verletzte als AS und AHB.

Hypothese m: IMT haben eine höhere Suizidrate als AS, nicht aber als AHB.

\section{Methode}

\section{Stichprobe}

Wir verglichen IMT mit AS und AHB in den USA, Großbritannien, Deutschland, Australien, Frankreich, Schweden und Kanada zwischen Januar 1999 und Juni 2017. Der Zeitraum wurde gewählt, da es ab 1999 bzw. dem Amoklauf in Columbine zu einer deutlichen Zunahme solcher Taten kam (Bondü, 2012) und IMT seit den 2000er 
Jahren zugenommen haben (Gruenewald et al., 2016). Wir berücksichtigen Taten in westlichen OECD-Ländern, in denen sowohl IMT als auch AS und/oder AHB stattgefunden hatten, um eine gute Vergleichbarkeit der Gruppen zu gewährleisten. Voraussetzung für die Berücksichtigung einer Tat war Tötungsabsicht, selbst wenn sie dahingehend nicht zum Erfolg führte.

Zur Recherche islamistisch motivierter Terrorakte wurden Forschungsliteratur und Datenbanken, Websites und Listen im Internet, die terroristische Taten dokumentieren, gesichtet (https://en.wikipedia.org/wiki/School_shooting, Global Terrorism Database (START, 2017a)). Taten wurden berücksichtigt, wenn sie der Definition von Terrorismus entsprachen und (1) beabsichtigt waren; (2) ein islamistisch motiviertes Ziel verfolgten; (3) die Täter bekannt waren; (4) symbolischen Charakter hatten (nicht nur den Opfern persönlich schaden sollten); (5) außerhalb der legitimen Kriegsführung und nach Völkerrecht zulässigen Parameter lagen (Verbot willentlicher Angriffe auf Nicht-Kombattanten).

Grundlage der Recherche von Amokläufen an Bildungseinrichtungen war der Datensatz von Bondü und Beier (2014). Aus diesem wurden alle Taten in den genannten Ländern im relevanten Zeitraum ausgewählt und durch weitere Fälle seit 2012 ergänzt (19 AS, 9 AHB). Es wurden nur Fälle einbezogen, bei denen sich ein Bezug zur Schule erkennen ließ und eine Tatplanung mit Tötungsabsicht vorlag. Taten von Personen, die keine (ehemaligen) Schüler der betroffenen Schule waren, wurden nicht einbezogen. Vorfälle an Primar- und Sekundarschulen (z.B. Grundschulen, High-Schools) wurden als AS definiert, Taten an Universitäten, Berufsschulen, Colleges usw. als AHB kategorisiert.

Es wurden 86 IMT ( $N=100$ Täter), 172 AS ( $N=182$ Täter) sowie 30 ( $N=31$ Täter) AHB recherchiert.

\section{Methodisches Vorgehen}

$\mathrm{Zu}$ allen Fällen wurden soweit möglich Tatzeit (Tag, Monat, Jahr, Wochentag, Uhrzeit, Jahreszeit), Tatort (Land, Stadt), Tatwaffe(n), Anzahl der verletzten und toten Opfer, Suizid des Täters, Alter, Geschlecht, Migrationshintergrund und Täterzahl recherchiert. Ein Migrationshintergrund lag vor, wenn der Täter oder mindestens ein Elternteil nicht die Staatsangehörigkeit des Tatlandes besaß (Statistisches Bundesamt, 2017). $\mathrm{Da}$ die Informationen über Medienberichte gesammelt wurden, deren Vertrauenswürdigkeit fraglich ist (Langman, 2009), wurden nicht-objektive Merkmale wie psychische Gesundheit, Tatmotive oder soziale Beziehungen der Täter nicht erfasst. Die Informationen wurden nach Möglichkeit durch unterschiedliche Quellen bestätigt.

Wegen multipler Gruppenvergleiche $(N=13)$ wurde das $\alpha$-Fehler-Niveau auf .0038 adjustiert. $\mathrm{Zu}$ vergleichenden Analysen wurden nonparametrische Verfahren verwendet: Kruskal-Wallis-Test $(H)$ bei drei Gruppen, Dunn-Bonferroni-Tests für nachfolgende paarweise Vergleiche. Bei gerichteten Hypothesen wurde der $p$-Wert halbiert. Bei kategorialen Daten wurde der $\chi^{2}$-Test verwendet. Unterschiedliche terroristische 
Taten des gleichen Täters $(N=11)$ wurden separat behandelt. Bei der Analyse der Tätereigenschaften wurde der Täter nur einmal gewertet.

\section{Ergebnisse}

\section{Tathäufigkeiten}

Entsprechend Hypothese a nahm die Zahl der AS seit 2009 ab, die der IMT vor allem seit 2011 zu. Bei den AHB ließen sich keine Veränderungen ausmachen (geringe Fallzahlen!).

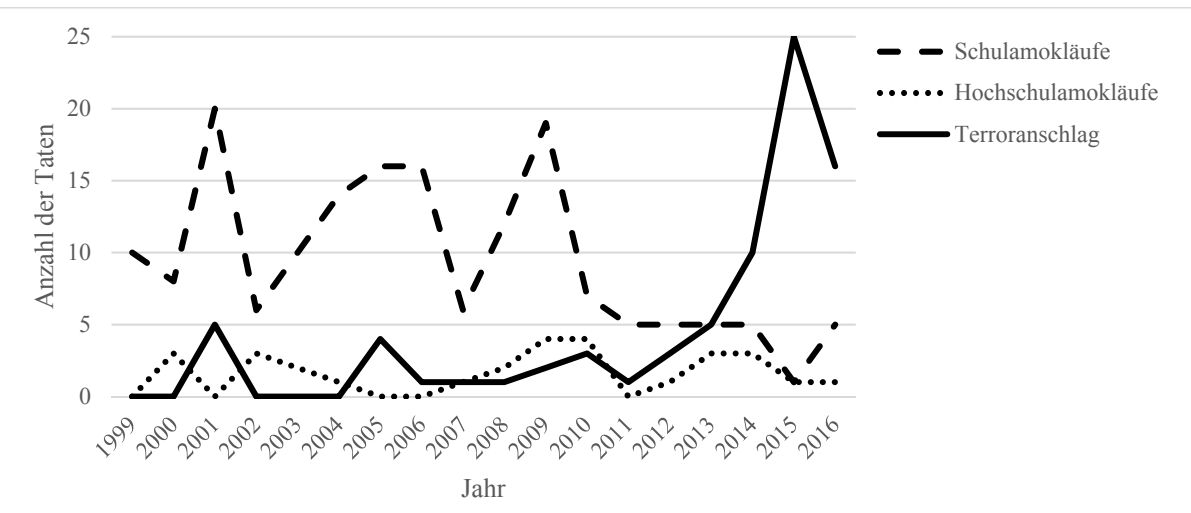

Abbildung 1. Anzabl der drei Tatgruppen in den betrachteten Ländern zwischen 1999 und 2016 (2017 nicht berücksichtigt, da nicht das vollständige Jabr erfasst wurde).

Tätermerkmale

Entsprechend Hypothese $c$ unterschieden sich AS $(N=182)$, AHB $(N=31)$ und IMT $(N=100)$ signifikant im Alter $(H(2)=188.19, p \leq .001, d=2.40)$. AS waren im Mittel 15.65 $(S D=2.47$, range $=10-32, M d n=15.0)$, AHB $28.45(S D=10.80$, range=15-62, $M d n=23.0)$ und IMT $26.13(S D=7.05$, range=15-52, $M d n=25.0)$ Jahre alt (Tabelle 1). AHB und IMT waren signifikant älter als AS (AHB: $z=-8.32, p \leq .001, d=1.39$; IMT: -12.55 , $p \leq .001, d=2.25$ ), unterschieden sich aber nicht signifikant voneinander.

Entsprechend Hypothese d, war der Anteil männlicher Täter in den drei Gruppen gleich hoch $\left(\mathrm{AS}=89 \%, \mathrm{AHB}=96,8 \%, \mathrm{IMT}=98 \% ; X^{2}(2)=8.59, p>.004\right)$.

Analog Hypothese e hatten IMT (87.9\%) signifikant häufiger einen Migrationshintergrund als AS $\left(8.9 \% ; X^{2}(1)=110.09, p \leq .001, d=1.60\right)$ und AHB $\left(34.5 \% ; X^{2}(1)=34.84\right.$, $p \leq .001, d=1.20)$. AHB hatten häufiger einen Migrationshintergrund als AS $\left(X^{2}(1)=10.50, p \leq .001, d=0.5\right)$. IMT waren hauptsächlich arabisch $(57.3 \%)$ und aus dem 
Nahen Osten (28.6\%), AS zu 70.8\% kaukasisch und aus den USA (50\%) oder Osteuropa (25.1\%) und AHB zu 40.7\% kaukasisch und zu 29.6\% asiatisch. Ihre Herkunftsländer lagen meist in Asien (43.8\%) oder sie stammten aus den USA (31.3\%). 36.7\% IMT führten ihre Tat in ihrem Geburtsland aus.

\section{Tatmerkmale}

Acht (4.7\%) Amokläufe an Schulen wurden von mehr als einem Täter (3x2 Täter, 4x3 Täter, $1 \times 4$ Täter), nur ein Amoklauf an einer höheren Bildungseinrichtung (3.3\%) wurde von zwei Tätern verübt. Terroristen führten in $22(25.6 \%)$ Fällen ihre Tat nicht al-

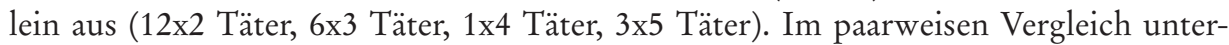
schieden sich AS nicht von AHB. AHB und IMT $(z=-3.40, p \leq .004, d=0.67)$ sowie AS und IMT $(z=-5.08, p \leq .001, d=0.67)$ unterschieden sich entsprechend Hypothese $f$ signifikant.

Wie in Hypothese g vermutet, zeigten die Häufigkeiten von AS und AHB ein U-förmiges Muster im Verlauf eines Jahres. Sie fanden ähnlich häufig im Frühling (MärzMai, AS=49 (28.5\%), AHB=11 (36.7\%)), Herbst (September-November, AS=47 $(27.3 \%), \mathrm{AHB}=7(23.3 \%))$ und Winter (Dezember-Februar, $\mathrm{AS}=57(33.1 \%), \mathrm{AHB}=9$ $(30 \%))$ statt, aber kaum im Sommer (Juni-August, AS=19 (11\%), AHB=3 (10\%)). IMT waren über das ganze Jahr verteilt. Die tatreichsten Monate waren Juni, Juli und Dezember (jeweils 11 Taten bzw. 12.8\%). Insgesamt wurden 9 der 86 Taten im Untersuchungszeitraum während des Ramadans verübt, davon alle seit 2015. Im Gruppenvergleich unterschieden sich IMT und AS signifikant in der Tatrate im Sommer $\left(X^{2}(1)=10.37, p \leq .001, d=0.41\right)$. AHB und IMT sowie AS und AHB zeigten keine Unterschiede.

Die meisten AS (42, 24.6\%) und AHB (9,30\%) fanden montags statt. Bei den IMT war Freitag der häufigste Tattag (19, 22.4\%). AS (166, 97.1\%) und AHB (29, 96.7\%) fanden überwiegend unter der Woche (Montag-Freitag) statt, IMT auch am Wochenende $(16,18.8 \%)$. Hypothese $h$ wird beibehalten. Der Unterschied zwischen den Gruppen war signifikant $\left(X^{2}(2)=21.11, p \leq .001, \varphi_{c}=0.27\right)$. AS fanden überwiegend in den frühen Morgenstunden (4-10 Uhr, 64 (53.8\%)) statt, AHB (9 (31\%)) meist am späteren Vormittag (10-12 Uhr). IMT fanden sowohl morgens (4-10 Uhr, 21 (25.6\%)), nachmittags (14-18 Uhr, 20 (24.4\%)), als auch abends (18-23 Uhr, 22 (26.8\%)) statt. Die Gruppenunterschiede waren signifikant $\left(X^{2}(12)=71.53, p \leq .001, \varphi_{\mathrm{c}}=0.39\right)$, Hypothese $i$ wird beibehalten.

Entsprechend Hypothese $b$ ereigneten sich die meisten AS $(N=135,78.5 \%)$ und AHB $(N=26,86.7 \%)$ innerhalb der hier betrachteten Länder in den USA, die meisten IMT in Frankreich und den USA ( $N=29,33.7 \%)$.

Schusswaffen waren gemäß Hypothese $j$, in allen Tätergruppen die häufigsten Tatwaffen (AS: $47.8 \%, N=88$; AHB: $63.6 \%, N=21$; T: 34.2, $N=38$ ), jeweils gefolgt von Klingenwaffen (AS: $42.9 \%, N=79$; AHB: $30.3 \%, N=10$; T: $34.2 \%, N=38$ ). IMT nutzten zudem häufiger explosive Stoffe $(26(23.4 \%))$ und Fahrzeuge $(12.6 \%, N=14)$. AHB 
nutzten signifikant häufiger Schusswaffen als IMT $\left(X^{2}(1)=9.09, p \leq .004, d=0.5\right)$. AS und AHB sowie AS und IMT unterschieden sich nicht. AS nutzten Klingenwaffen signifikant häufiger als IMT $\left(X^{2}(1)=9.39, p \leq .001, d=0.4\right)$. Zwischen AHB und IMT sowie AS und AHB gab es keine Unterschiede. Bomben fanden bei AS (10 (5.4\%)) und AHB ( 0 $(0 \%))$ selten bzw. nie Verwendung. Der Unterschied zu den IMT war entsprechend Hypothese $k$ signifikant $\left(X^{2}(2)=27.62, p \leq .001, \varphi_{c}=0.29\right)$.

Durch AS wurden 130 (27.5\%) Menschen getötet und 343 (72.5\%) verletzt, durch AHB 85 (36.8\%) Personen getötet und 146 (63\%) verletzt. Bei den IMT wurden 3411 (60.4\%) Menschen getötet und 2233 (39.6\%) verletzt. Den größten Anteil an den Toten hatten die Anschläge am 11.9.2001 mit 2754 Toten (65,46\%, k.A. zu Verletzten). IMT forderten signifikant mehr Tote $(M=39.66, S D=208.92, M d n=1.0)$ als AS $(M=0.76$, $S D=2.92, M d n=0.00 ; z=-5.91, p \leq .001, d=0.79)$ und AHB $(M=2.83, S D=5.91, M d n=1.0$; $z=-5.36, p \leq .001, d=0.81)$. Zwischen AHB und IMT bestand kein Unterschied. Wurden die Terroranschläge vom 11.9.2001 ausgeschlossen, unterschieden sich IMT $(M=5.29$, $S D=14.94, M d n=1.0)$ und AS weiter signifikant $(z=-5.38, p \leq .001, d=0.71)$, nicht aber IMT und AHB. IMT forderten signifikant mehr Verletzte $(M=26.58, S D=69.04$, $M d n=2.0)$ als AS $(M=2.04, S D=4.22, M d n=1.0 ; z=-3.17, p \leq .004, d=0.40)$. AS und AHB sowie AHB und IMT unterschieden sich nicht. Hypothese $l$ wird teilweise beibehalten.

AS $(22(13.3 \%))$ und IMT (34 (34\%)) unterschieden sich in der Suizidrate $\left(X^{2}(1)=16.16, p \leq .001, d=0.49\right)$, nicht jedoch AS und AHB (10 (32.3\%)) sowie AHB und IMT. Hypothese $m$ wird beibehalten. Der häufigste Tatausgang bei IMT war Erschießen durch die Polizei (36 (36\%)), gefolgt von Suizid (34 (34\%)) und Festnahmen (29 (29\%)). Bei AS war der häufigste Tatausgang die Festnahme des Täters (140 $(83.8 \%))$. Bei AHB war der Tatausgang meist Festnahme (18 (58.1\%)), gefolgt von Suizid $(10(32.3 \%))$ und Erschießen durch die Polizei (6.5\% (2)). Der Gruppenunterschied war signifikant $\left(X^{2}(8)=104.99, p \leq .001, \varphi_{c}=0.42\right)$. 


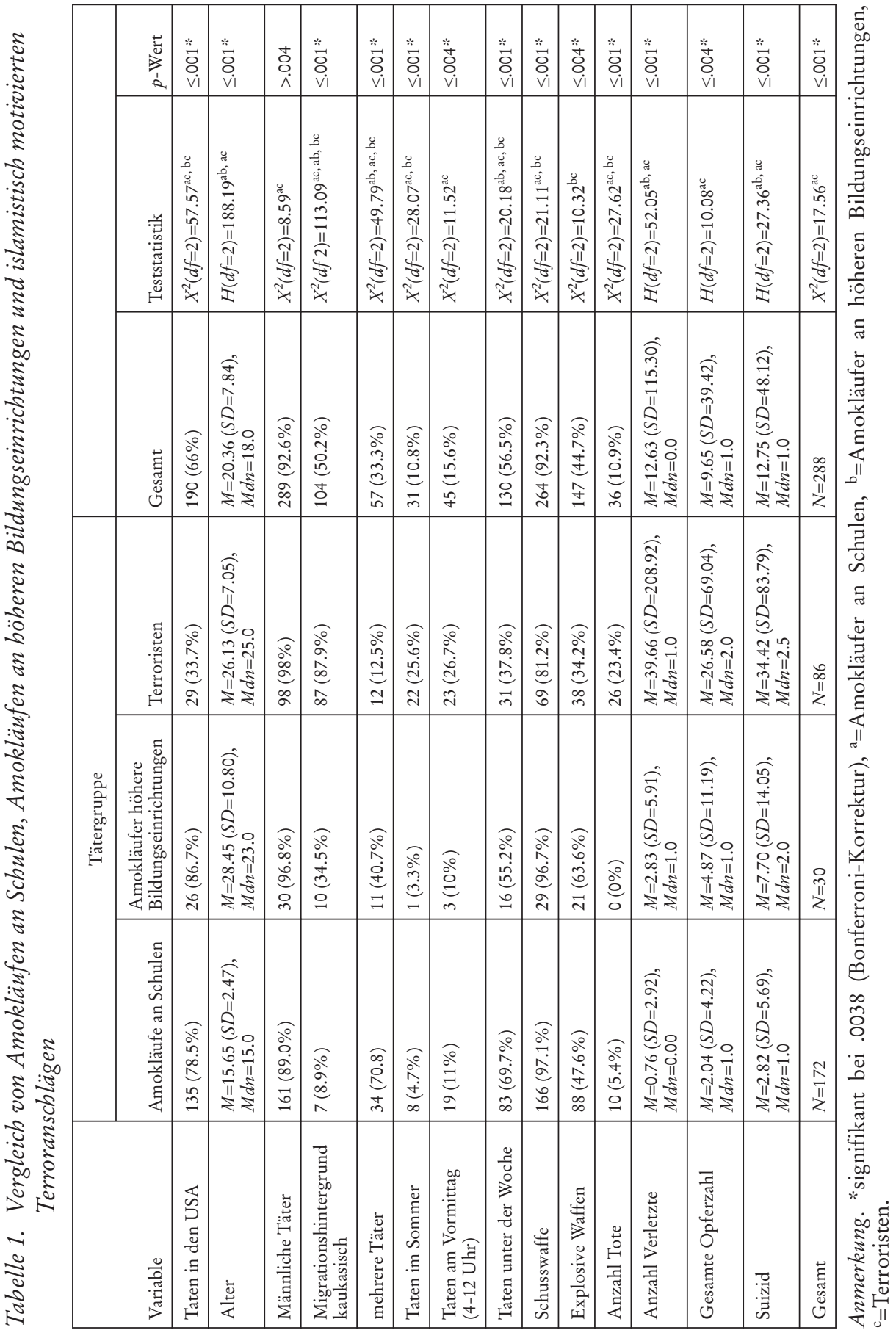




\section{Diskussion}

\section{Häufigkeiten}

Es wurden mehr AS als AHB und IMT ermittelt. Die Zahl der jährlichen Amokläufe im Bildungskontext nahm über den Erhebungszeitraum ab, die der Terroranschläge zu. Unter den ausgewählten Ländern fanden die meisten Taten in den USA statt. Ein möglicher Grund für häufige Taten in den USA sind liberale Waffengesetze und die hohe Präsenz von Schusswaffen im Alltag (Bondü, 2012). Der Anstieg von IMT erklärt sich vermutlich unter anderem durch den Aufruf des IS 2014 zum Kampf gegen „Ungläubige“ (Neumann, 2016). Da nur in den Medien berichtete Fälle berücksichtigt wurden, kann auch unterschiedliche Aufmerksamkeit der Medien die Anzahl der recherchierbaren Taten beeinflusst haben. Medien richten ihr Augenmerk besonders auf Taten, die mit vielen Opfern enden, in den letzten Jahren also insbesondere terroristische Taten. Daher sind diese Fälle in Medienberichten und somit dieser Studie im Vergleich zu Taten mit geringeren Opferzahlen, wie bei AS häufiger der Fall, womöglich überrepräsentiert. Nicht berücksichtigt wurden zudem Länder wie die Türkei, in denen viele Terrorakte vorkommen, die Übertragbarkeit von Befunden auf westliche Länder aber fraglich ist (hier sind häufig Muslime selbst von den Taten betroffen).

\section{Tätermerkmale}

Wie erwartet unterschieden sich die Tätergruppen im Alter. Das Alter von IMT und AHB war vergleichbar. Der hohe Altersschnitt von AHB stützt die Vermutung, dass Leistungsversagen eine Rolle bei diesen Taten spielen könnte (z.B. häufigere Wiederholungen von Prüfungen; Newman \& Fox, 2009). Die Attribution eigenen Versagens auf externale Ursachen wäre demnach eine Möglichkeit des Selbstschutzes. Allerdings könnten sich Amokläufer, die schon lange eine Tat planen, auch weniger Mühe im Bildungskontext geben (Bondü \& Beier, 2014). Die große Altersspanne zeigt, dass islamistisch motivierter Terror überwiegend, aber nicht nur, von jungen Tätern ausgeübt wird. Ältere Täter sind zudem oft Einzeltäter (Gill, Horgan \& Deckert, 2013). Möglicherweise spiegelt das Alter der Täter daher Unterschiede in den Motiven. Bei jüngeren Tätern wird vermutet, dass sie sich aufgrund des Drucks von Freunden und Bekannten einer Terrororganisation anschließen und dann radikalisiert werden; ältere Täter agieren womöglich aufgrund bestehender extremistischer Auffassungen, die durch islamistische Propaganda verstärkt wurden (Neumann, 2016). Altersunterschiede erklären ggf. auch weitere Gruppenunterschiede (Bondü \& Beier, 2014): Beispielsweise können ältere Täter leichter legal Schusswaffen erwerben (s.u.), die wiederum häufiger zu höheren Opferzahlen führen als Klingen- oder stumpfe Waffen.

Erwartungsgemäß waren männliche Täter in allen Gruppen stark überrepräsentiert. Vermutlich gibt es unter den IMT aufgrund der traditionell männlichen Ausrichtung des Dschihads kaum Frauen (Neumann, 2016). Aus einigen arabischen und afrikanischen Ländern (z.B. Palästina; Mali) sind jedoch islamistische Täterinnen bekannt, die 
aufgrund der Auswahl der Tatländer nicht in der Stichprobe berücksichtigt wurden. Daher sind Frauen hier womöglich unterrepräsentiert. Ferner sind männliche Täter mit zunehmender Deliktschwere grundsätzlich überrepräsentiert (Gottfredson \& Hirschi, 1990). Ausgehend vom vorliegenden Befund, dass IMT im Mittel mehr Opfer fordern als AS, könnte auch dies den größeren Unterschied in der Geschlechterverteilung erklären.

Wie erwartet hatten IMT häufiger einen Migrationshintergrund als Amokläufer im Bildungskontext. Dies ist kaum verwunderlich: Erstens stammten einige Täter ursprünglich aus islamischen Ländern, hatten sich dort radikalisiert und waren zum Zweck der Tat(planung) in ein westliches Land eingereist. Zweitens werden Personen mit muslimischem Hintergrund durch islamistische Propaganda einfacher erreicht als nicht-muslimische Personen, denen der entsprechende Zugang fehlt. So verübten mehr als ein Drittel der recherchierten IMT die Tat in ihrem Geburtsland (Roy, 2007). Bedenkt man, dass die Richtung der Radikalisierung scheinbar auch von der verfügbaren und leicht zugänglichen Ideologie abhängt (Neumann, 2017), erscheint die häufigere Radikalisierung von Menschen mit Migrationshintergrund im islamistischen Kontext folgerichtig. Personen ohne Migrationshintergrund sollten eher für christlich-radikale, links- oder rechtsextreme Ideologien ansprechbar sein. Das Beispiel von Konvertiten unter den Tätern zeigt allerdings, dass natürlich auch andere Personengruppen islamistisch radikalisiert werden können. Drittens sind Erfahrungen von Benachteiligung (z.B. Diskriminierung), die Personen mit Migrationshintergrund häufig betreffen, ein Nährboden für Radikalisierung. Viertens könnten Kriegs- und Migrationserfahrungen und deren Folgen eine psychische Destabilisierung bedingen und vermeintliche Gründe für die Tatrechtfertigung liefern.

AS haben selten einen Migrationshintergrund und sind entsprechend der in den westlichen Ländern vorherrschenden independenten Kultur stärker individuell motiviert. Der Anteil des Migrationshintergrunds zwischen AHB und IMT unterschied sich nicht, aber die typischen Herkunftsländer: AHB stammten überproportional häufig aus asiatischen Ländern. Hier ist zu vermuten, dass Migrationserfahrungen, stärkerer Leistungsdruck, kulturelle Skripts, die Ehre betonen und Suizid begünstigen (Newman \& Fox, 2009; Shon, 2011), die Dekompensation und die Taten beeinflusst haben könnten. Der kulturelle Hintergrund der Täter kann somit die Tatmotive und -ausgestaltung prägen.

AS begingen ihre Taten häufiger allein als IMT, vermutlich aufgrund verschiedener Motive. Terroristen sind häufig in ein soziales System eingebunden, dessen Mitglieder die Ideologie teilen; Amokläufer an Bildungseinrichtungen sind meist Einzeltäter, die ihre Taten idiosynkratisch (teilweise unter Rückgriff auf frühere Täter und bestehende Ideologien) begründen (Bannenberg, 2017; Bondü \& Beier, 2014). Ferner sind IMT schwieriger zu planen als AS, da oft Großveranstaltungen anvisiert werden (Urban, 2006). Hier bieten mehrere Personen, die an der Tatplanung und -umsetzung beteiligt sind, eine größere Erfolgschance. 


\section{Tatmerkmale}

Die Häufigkeit von Amokläufen an Bildungseinrichtungen wies ein U-förmiges Muster im Jahresverlauf mit einem Rückgang in den Sommermonaten auf, der sich durch die Sommerferien erklären lässt. IMT waren über das Jahr weitgehend gleichverteilt. Mit neun Taten während des Ramadan zeigte sich insgesamt gesehen keine besondere Häufung in diesem Zeitraum. Allerdings ist auffällig, dass alle Taten während des Ramadans seit 2015 auftraten, was auf mögliche Veränderungen in diesem Tatmerkmal hindeuten könnte. Da terroristische Taten praktisch überall im öffentlichen Raum ausgeführt werden können und vielfältige Tatgelegenheiten bestehen, gibt es für diese kaum Beschränkungen. Dies erklärt auch, warum Amokläufe in Bildungseinrichtungen meist unter der Woche in den Morgenstunden stattfanden (Unterrichtszeiten), IMT hingegen auch am Wochenende und über den Tag verteilt. Der häufigste Tattag war Freitag, der wichtigste Wochentag in der muslimischen Kultur mit religiös-symbolischer Bedeutung. IMT führen ihre Taten zudem vermutlich gerade am Wochenende mit Großveranstaltungen durch, bei denen viele potenzielle Opfer zusammenkommen (Urban, 2006). Taten unter der Woche erfahren wiederum womöglich hohe Aufmerksamkeit, da die Bevölkerung aus ihrem Alltag gerissen wird und somit das Gefühl der Unsicherheit und Unkontrollierbarkeit besonders stark ist.

In allen Gruppen waren Schusswaffen die häufigsten, Klingenwaffen die zweithäufigsten Tatwerkzeuge. AHB verwendeten Schusswaffen signifikant häufiger als IMT, vermutlich weil diese daneben öfter zusätzlich explosive Stoffe und Kraftfahrzeuge nutzten. Die Verwendung unterschiedlicher Tatwaffen kann mit dem Aufruf des IS zusammenhängen, den Kampf gegen „Ungläubige“ mit allen erdenklichen und verfügbaren Mitteln voranzutreiben (Neumann, 2016). Klingenwaffen sind leicht verfügbar und auch für jüngere Personen leicht zugänglich, sodass sie vermutlich daher von AS häufiger benutzt wurden als den beiden anderen Gruppen.

Vermutlich aufgrund der Vielzahl an Waffen, des Einsatzes von explosiven Stoffen und des Ziels, möglichst viele Ungläubige zu töten (Nedopil, 2014), wurden bei IMT im Mittel mehr Menschen getötet und verletzt als bei AS. AHB töteten und verletzten vermutlich aufgrund der häufigeren Nutzung von Schusswaffen mehr Menschen als AS. Meloy et al. (2004) vermuten zudem, dass AS aufgrund ihres jungen Alters mehr Hemmungen haben, andere zu töten.

Wie angenommen suizidierten sich IMT und AHB häufiger als AS. Möglicherweise können sich jugendliche Täter aufgrund ihrer Unerfahrenheit mit Gewalt und Tod häufiger nicht überwinden, sich zu töten (Meloy et al., 2004). Auch häufigere psychische Störungen bei älteren Tätern könnten Suizid begünstigen (Newman \& Fox, 2009). Ferner zeigte sich, dass sich AS umso häufiger suizidierten, je höher die Zahl der Tatopfer war (Bondü, 2012). Dies spiegelt sich auch in den vorliegenden Ergebnissen, nach denen die Opferzahlen bei IMT und AHB signifikant höher waren als bei AS. Während diese Suizid meist als Ausweg aus der Hoffnungslosigkeit sehen (Vossekuil et al., 2002), spielt der Suizid bei IMT als Versinnbildlichung des Märtyrertums eine Rol- 
le. Der Tod wird in der Hoffnung, durch die Tat ins Paradies einzugehen, positiv bewertet (Abdel-Khalek, 2004).

Amokläufer an Bildungseinrichtungen und IMT zeigen somit sowohl Unterschiede als auch Gemeinsamkeiten. Viele Unterschiede scheinen auf situative Einflüsse (Tatgelegenheit) sowie das Alter der Täter (z.B. Zugang zu Waffen, Opferzahl) zurückzugehen. Entsprechend zeigt unsere Studie, dass sich insbesondere IMT und AHB ähneln. Dies erklärt, warum Amokläufer und terroristische Einzeltäter teilweise zusammengefasst wurden (Leuschner, 2013). Allerdings ist zu bedenken, dass sich in beiden Gruppen sowohl Einzel- als auch Gruppentäter fanden und die vorliegende Analyse auch Unterschiede zwischen den Tätergruppen zeigte. So könnte es sein, dass die Art der Tat insbesondere bei Einzeltätern primär davon abhängt, ob sie mit einer bereits bestehenden Ideologie in Kontakt kommen.

\section{Limitationen und Ausblick}

Unsere Studie verglich systematisch eine große Anzahl von AS, AHB und IMT in westlichen Ländern. Bei der Interpretation der Ergebnisse sind jedoch auch Einschränkungen zu berücksichtigen: Medienberichte beinhalten häufig stereotype und verzerrte Informationen (Muschert \& Larkin, 2007), daher konzentrierte sich die Analyse auf objektive Tat- und Tätermerkmale und wurden weitere potenziell interessante Merkmale (z.B. psychische Störungen, Auslöser, Tatmotive) nicht berücksichtigt. Über Medien können nur Taten recherchiert werden, über die berichtet wird, und nur Merkmale erhoben werden, zu denen Informationen vorliegen. Fehlende Informationen zu einzelnen Merkmalen verringerten daher die Stichprobengröße in verschiedenen Analysen. Insbesondere bei Fällen ohne Tote, mit wenigen Verletzten oder mit jungen Tätern (Bondü, 2012) waren oft nur wenige Informationen verfügbar. Da die Taten überwiegend in den USA stattfanden, können die Resultate nicht uneingeschränkt übertragen werden. So ist zu vermuten, dass sich nicht nur die Motivation für terroristische Taten zwischen westlichen und arabischen sowie anderen Ländern unterscheiden könnte, sondern damit zusammenhängend auch die in der vorliegenden Studie betrachteten Tat- und Tätermerkmale.

Zukünftige Forschung sollte die Merkmale verschiedener Gruppen von Massenmördern auf der Grundlage von Aktenanalysen untersuchen, die auch Aufschlüsse über personale Risikofaktoren (z.B. psychische Probleme), Warnsignale und Tatmotive geben können (Bannenberg, 2017; Böckler et al., 2018). Zudem sollten potentielle Subgruppen wie beispielsweise Einzel- und Gruppentäter oder eingereiste im Vergleich zu in den betroffenen Ländern aufgewachsene Täter innerhalb der islamistisch motivierten Täter identifiziert werden, die ggf. unterschiedliche Präventions- und Interventionsmaßnahmen erfordern. 


\section{Literatur}

Abdel-Khalek, A.M. (2004). Neither altruistic suicide, nor terrorism but martyrdom: A Muslim perspective. Archives of Suicide Research, 8, 99-113.

Bakker, E. (2011). Characteristics of jihadi terrorists in Europe. In Coolsaet, R. (Eds.), Jihadi Terrorism and the Radicalisation Challenge. European and American Experiences (p. 131-144). Aldershot: Ashgate.

Bannenberg, B. (2017). Schlussbericht Projekt TARGET. Teilprojekt Gießen: Kriminologische Analyse von Amoktaten - junge und erwachsene Täter von Amoktaten, Amokdrobungen im Verbundprojekt TARGET. Verfügbar unter: http://www.fv-sggs. de/tl_files/doc/Schlussbericht_Target_Giessen.pdf [Zugriff am 10.5.2017]

Böckler, N., Leuschner, V., Zick, A., \& Scheithauer, H. (2018). Same but Different? Developmental Pathways to Demonstrative Targeted Attacks - Qualitative Case Analyses of Adolescent and Young Adult Perpetrators of Targeted School Attacks and Jihadi Terrorist Attacks in Germany. International Journal of Developmental Science, 12, $5-24$.

Böckler, N., Seeger, T., Sitzer, P., \& Heitmeyer, W. (Eds.). (2012). School Shootings: International Research, Case Studies, and Concepts for Prevention. New York: Springer.

Bondü, R. (2012). School Shootings in Deutschland. Internationaler Vergleich, Warnsignale, Risikofaktoren, Entwicklungsverläufe. Dissertationsschrift. Berlin. Verfügbar unter: http://www.diss.fu-berlin.de/diss/receive/FUDISS_thesis_000000037683 [Zugriff am 27.12.2017]

Bondü, R. \& Beier, S. (2014). Two of a kind? Differences and similarities of attacks in schools and in institutes of higher education. Journal of Interpersonal Violence, 30, 253-271.

Borum, R. (2003). Understanding the terrorist mind-set. FBI L. Enforcement Bull., 72.

Capellan, J.A., Johnson, J., Porter, J. R., \& Martin, C. (2018). Disaggregating mass public shootings: a comparative analysis of disgruntled employee, school, ideologically motivated, and rampage shooters. Journal of Forensic Sciences. Online first: https:// onlinelibrary.wiley.com/doi/full/10.1111/1556-4029.13985

Gill, P., Horgan, J., \& Deckert, P. (2013). Bombing alone: Tracing the motivations and antecedent behaviors of lone-actor terrorists. Journal of Forensic Sciences, 59, 425-435.

Gottfredson, M. R. \& Hirschi, T. (1990). A general theory of crime. Standford: Stanford University Press.

Gruenewald, J., Klein, B.R., Freilich, J.D., \& Chermak, S. (2016). American jihadi terrorism: A comparison of homicides and unsuccessful plots. Terrorism and Political Violence, 1-20.

Institute for Economics and Peace. (2016). Global Terrorism Index 2016. Verfügbar unter: http://economicsandpeace.org/reports/ [Zugriff am 22.4.2017]

RPsych 5. Jg. 2/2019 
Langman, P. (2009). Amok im Kopf. Warum Schüler töten. Weinheim: Beltz.

Leuschner, V. (2013). Exzessive individuelle Gewalt. „School Shootings“ und „Lone Wolf Terrorism“ als soziale Phänomene. Berliner Journal für Soziologie, 23, 27-49.

Liem, M., van Buuren, J., de Roy van Zuijdewijn, J., Schönberger, H., \& Bakker, E. (2018). European Lone Actor Terrorists Versus "Common" Homicide Offenders: An Empirical Analysis. Homicide Studies, 22, 45-69.

McCauley, C., Moskalenko, S., \& Van Son, B. (2013). Characteristics of lone-wolf violent offenders: A comparison of assassins and school attackers. Perspectives on Terrorism, 7, 4-24.

Meloy, J.R., Hempel, A.G., Gray, B.T., Mohandie, K., Shiva, A., \& Richards, T.C. (2004). A comparative analysis of North American adolescent and adult mass murderers. Behavioral Sciences E the Law, 22, 291-309.

Miller, E., Kane, S., Kammerer, W., \& Wingenroth, B. (2016). Patterns of Islamic Staterelated Terrorism, 2002-2015. START.

Muschert, G.W. \& Larkin, R.W. (2007). The Columbine High School shootings. In F. Bailey \& S. Chermak (Eds.), Crimes \& Trials of the century (S. 253-266). Westport: Praeger Publishers.

National Consortium for the Study of Terrorism and Responses to Terrorism (START). (2016). Annex of Statistical Information. Country Reports on Terrorism 2015. Verfügbar unter: https://www.state.gov/j/ct/rls/crt/2015/257526.html [Zugriff am 23.7.2017]

National Consortium for the Study of Terrorism and Responses to Terrorism (START).

(2017a). Global Terrorism Database [Datenbank]. Verfügbar unter: https://www.start. umd.edu/gtd [Zugriff am 1.5.2017]

National Consortium for the Study of Terrorism and Responses to Terrorism (START).

(2017b). Codebook: Inclusion criteria and variables. Verfügbar unter: http:// www.start.umd.edu/gtd/downloads/Codebook.pdf [Zugriff am 23.6.2017]

Nedopil, N. (2014). Gekränkte Eitelkeiten. Forensische Psychiatrie, Psychologie, Kriminologie, 8, 246-253.

Neumann, P.R. (2016). Der Terror ist unter uns. Dschibadismus und Radikalisierung in Europa. Berlin: Ullstein Buchverlage.

Newman, K. \& Fox, C. (2009). Repeat tragedy: Rampage shootings in American high school and college settings. American Behavioral Scientist, 52, 1286-1308.

Roy, O. (2007). Islamic terrorist radicalisation in Europe. European Islam: Challenges for society and public policy, 52-60. 
Shon, P.C.H. (2011). "Asian really don't do this": On-scene offense characteristics of Asian American school shooters, 91-07. Asian Criminology, 7, 252-271.

Statistisches Bundesamt (2017). Bevölkerung und Erwerbstätigkeit. Bevölkerung mit Migrationshintergrund. Ergebnisse des Mikrozensus 2015. Verfügbar unter: https://ww w.destatis.de/DE/Publikationen/Thematisch/Bevoelkerung/MigrationIntegration/Mig rationshintergrund 2010220157004.pdf?_blob=publicationFile [Zugriff am 13.3.2019] Urban, J. (2006). Die Bekämpfung des internationalen islamistischen Terrorismus. Wiesbaden: Verlag für Sozialwissenschaften.

Vossekuil, B., Fein, R. A., Reddy, M., Borum, R., \& Modzeleski, W. (2002). The Final Report and Findings of the Safe School Initiative: Implications for the Prevention of School Attacks in the United States. Washington: U.S. Secret Service and U.S. Department of Education.

\section{Kontakt:}

Rebecca Bondü (Corresponding author)

Psychologische Hochschule Berlin

Universität Konstanz

Am Köllnischen Park 2

10179 Berlin

Email: r.bondue@phb.de.

Laura Tampe, Psychologische Hochschule Berlin.

Katharina Menke, Universität Konstanz. 